\title{
Prevalence of visual snow and relation to attentional absorption
}

\author{
Rui Miguel Costa \\ William James Center for Research, ISPA - Instituto Universitário, Lisbon, Portugal \\ Pedro Campos \\ ISPA - Instituto Universitário, Lisbon, Portugal \\ Madalena Wiborg \\ ISPA - Instituto Universitário, Lisbon, Portugal \\ Catarina Rebôlo \\ ISPA - Instituto Universitário, Lisbon, Portugal \\ Marc Wittmann \\ Institute for Frontier Areas of Psychology and Mental Health, Freiburg, Germany \\ Jürgen Kornmeier \\ Institute for Frontier Areas of Psychology and Mental Health, Freiburg, Germany
}

\section{Corresponding author}

Rui Miguel Costa

ISPA - Instituto Universitário, Rua do Jardim do Tabaco 34, 1149-041, Lisbon, Portugal.

Email: rcosta@ ispa.pt 


\title{
Prevalence of visual snow and relation to attentional absorption
}

\begin{abstract}
Visual snow is a condition of unclear prevalence characterized by tiny flickering dots throughout the entire visual field. It appears to result from visual cortex hyperactivity and possibly correlates with propensity to be engrossed in sensory and imaginary experiences (absorption). The prevalence and correlates of visual snow, and emotional reactions to it, were explored in the general Portuguese population with three studies with online surveys. In Study 1, 564 participants were shown an animated graphic simulation of visual snow and asked to rate how frequently they see it on a scale anchored by $0 \%$ and $100 \%$ of the time. They also reported their degree of distress and fascination resulting from visual snow. Absorption was measured with the Modified Tellegen Absorption Scale. 44\% of respondents reported they see visual snow at least $10 \%$ of the time, and $20 \%$ reported seeing it between $80 \%$ and $100 \%$ of the time. Similar to findings in clinical samples, the frequency of visual snow correlated with tinnitus frequency, migraine, and entoptic phenomena, but not with ophthalmologic problems. It was confirmed that visual snow is related to absorption. Although distress caused by visual snow was generally absent or minimal, a substantial minority (28\%) reported moderate to high levels of distress. High fascination with visual snow was reported by $9 \%$. In Studies 2 and 3, visual snow was measured by means of verbal descriptions without graphic simulation ("visual field full of tiny dots of light" and "world seen with many dots of light", respectively). The results were similar to those in Study 1, but seeing visual snow $80 \%-100 \%$ of the time was less frequent $(6.5 \%$ in Study 2 and 3.6\% in Study 3). Visual snow has been insufficiently investigated. More research is needed to uncover underlying neurophysiological mechanisms and psychological and behavioral correlates.
\end{abstract}




\section{Introduction}

Visual snow refers to a visual phenomenon characterized by tiny flickering dots occupying the entire visual field. The dots are often described as bright on dark backgrounds and dark on light backgrounds. They can also be described as brighter than the background or colorful [1-3]. Visual snow has attracted much attention in recent years [1-16], often with the implication that it is a pathological and rare condition. The term "visual snow syndrome" was proposed when it occurs with other visual phenomena, like palinopsia (after-images or visual trailing), photophobia (oversensitivity to light), nyctalopia (night-blindness), and entoptic phenomena (visual phenomena arising with sources in the eye [1-3]. Visual snow appears to be unrelated to ophthalmologic problems, but co-occurs frequently with migraine and tinnitus [2$7,12]$.

Most research on visual snow has been conducted with clinical samples. A subgroup of persons experiencing the visual snow phenomenon may be worried about it and seek clinical assessment of potentially serious causes. Only those patients then typically enter the clinical studies. Many people experiencing visual snow may not be worried by this experience, as indicated by numerous anecdotal reports on the internet. Even some people in clinical studies are not worried about it [17]. One study examined the prevalence and correlates in the general population. In a representative British sample, $3.7 \%$ of the respondents reported seeing visual snow (CI: $2.7-5.2$ ), and 2.2\% fulfilled the criteria for the visual snow syndrome (CI: $1.4-3.3$ ) [18]. Respondents in this nonclinical representative sample who met the criteria for the visual snow syndrome were more likely to report severe headaches, tinnitus, and mood symptoms [18], which is congruent with the studies in clinical samples. The obtained prevalence of visual snow may be underestimated, as the study asked whether respondents experience visual snow "always 
or almost always". Visual snow may be a transient experience or even a natural phenomenon which many people perceive if attention is focused on it [19]. Herein, we present the results of three studies examining the prevalence and correlates of visual snow in the general Portuguese population. We also wanted to find out to what extent visual snow causes not only distress, but also fascination, because many people are fascinated by particular peculiar visual phenomena.

\section{Attentional absorption and visual snow}

An untested possibility is that people experiencing visual snow may be more engrossed in perceptual and imaginary experiences, because visual snow seems to result from hyperexcitability of the visual cortex [4-7,9-11]. Such hyperexcitability intensifies visual perceptions and mental imagery, thereby facilitating attentional absorption in percepts and mental images. Research confirms that visual snow is associated with intensified sensory experiences [20]. Visual snow has also been associated with greater activity [5,7,9] and greater gray matter volume of the lingual gyrus [15], an area of the associative visual cortex that tends to be larger in people with greater ability to imagine [21], and that tends to be more active during tasks of internally directed attention [22] and mental imagery [23]. Such increased activity of the visual cortex is thought to facilitate the awareness of visual snow, which might result from the conscious perceptions of a particular form of sensory information that normally remains subthreshold [5].

The individual differences in the propensity to be attentionally engrossed in sensory and imaginary experiences are reflected in the personality trait "absorption" [24-30]. This trait refers to the tendency to experience absorbed attentional states, i. e., intense states of focused, nonreflective, attention to perceptual or imaginary experiences [24-30]. Tellegen and Atkinson 
(1974) reported three overlapping aspects of absorbed states: 1) a heightened sense of reality of the attentional object that to some extent excludes metacognitions (thinking about the attentional object), 2) imperviousness to normally distracting events as individuals become less aware of the surrounding space, and 3) an altered sense of reality. The experience of one part of reality is amplified, while the presence of other aspects, not only the environment, but also thoughts and characteristics of the self, retreat in consciousness. In retrospect, the experience might be remembered as fascinatingly strange or intriguing, or even as a discontinuity of the usual awareness of self and the world. A great sense of proximity to the attentional object is felt. The type of focused attention that characterizes absorption is not the result of sustaining attention actively and with conscious effort; instead, absorbed states "have the quality of effortlessness, as if they happened by themselves, and in that sense, of involuntariness" (p. 222) [27]. Fascination is another term for the experience of absorption [24].

Higher propensity to experience absorbed states has been associated with openness to experience [28,31-33], appreciation of arts [33], rich and vivid imaginary [34,35], daydreaming as means to problem solving [36], greater hypnotic susceptibility [24-26,32,34,36], higher sexual desire [28,31], and mystical experiences [25,37]. People with the propensity for absorption are more prone to unusual perceptual experiences [29]. Also, the scales commonly used to measure trait absorption have an item that taps into whether people experience after-images [24, 26], which is a criterion for the proposed visual snow syndrome [1-3]. The experience of visual snow may well be related to the personality trait of absorption, but the possibility of such relationship was never examined.

To summarize, in the present study, we explored how frequently visual snow occurs in an unselected nonclinical group, and how frequently visual snow causes distress and fascination in 
this group. We also explored correlations between the frequency of seeing visual snow and tinnitus, migraine, and trait absorption.

\section{Method}

\section{Procedure}

Three studies on these research questions were performed in Portugal with online surveys advertised on social media between 2017 and 2021. These were part of a series of studies on correlates of attentional absorption. All studies received ethical approval from the local Ethics Committee. We will refer to the three studies as Study 1, Study 2, and Study 3. As described below, measures of visual snow differed across the studies because we were interested in comparing the prevalence of visual snow when visual snow is measured with the aid of an animated graphic simulation or by mere verbal descriptions. Table 1 illustrates the variables assessed in the three studies. Measures are described in the next subsection.

Table 1. Measures used in Studies 1, 2, and 3.

\begin{tabular}{|c|c|c|c|}
\hline & Study 1 & Study 2 & Study 3 \\
\hline $\begin{array}{l}\text { Visual snow assessed with the aid of animated } \\
\text { graphic simulation (seen with open eyes) }\end{array}$ & $\mathrm{X}$ & & \\
\hline $\begin{array}{l}\text { Visual snow assessed with the aid of animated } \\
\text { graphic simulation (seen with closed eyes) }\end{array}$ & $\mathrm{X}$ & & \\
\hline $\begin{array}{l}\text { Visual snow described as a visual field full of } \\
\text { tiny dots of light (seen with open eyes) }\end{array}$ & & $\mathrm{X}$ & \\
\hline Visual snow described as a visual field full of & & $\bar{X}$ & \\
\hline
\end{tabular}




\begin{tabular}{|c|c|c|c|}
\hline tiny dots of light (seen with closed eyes) & & & \\
\hline $\begin{array}{l}\text { Visual snow described as the world seen with } \\
\text { many dots of light (seen with open eyes) }\end{array}$ & & & $\mathrm{X}$ \\
\hline Absorption & $\bar{X}$ & $\mathrm{X}$ & $\mathrm{X}$ \\
\hline $\begin{array}{l}\text { Blue field entoptic phenomena assessed with } \\
\text { the aid of animated graphic simulation }\end{array}$ & $\mathrm{X}$ & & \\
\hline Floaters assessed with the aid of pictures & $\mathrm{X}$ & & \\
\hline Distress caused by visual snow & $\mathrm{X}$ & $\bar{X}$ & \\
\hline Fascination with visual snow & $\mathrm{X}$ & $\mathrm{X}$ & \\
\hline Tinnitus & $\mathrm{X}$ & $\mathrm{X}$ & \\
\hline Distress caused by tinnitus & $\mathrm{X}$ & $\mathrm{X}$ & \\
\hline Fascination with tinnitus & $\mathrm{X}$ & $\mathrm{X}$ & \\
\hline Situations triggering visual snow & $\bar{X}$ & $\mathrm{X}$ & \\
\hline $\begin{array}{l}\text { Life events associated with the first-time } \\
\text { appearance of visual snow }\end{array}$ & $\bar{X}$ & $\mathrm{X}$ & \\
\hline Analogies of visual snow & $\bar{X}$ & $\mathrm{X}$ & \\
\hline Time of visual snow first appearance & $\bar{X}$ & $\mathrm{X}$ & \\
\hline Intensification of visual snow over time & $\bar{X}$ & $\mathrm{X}$ & \\
\hline Computer-screen time & $\bar{X}$ & $\mathrm{X}$ & \\
\hline Migraine & $\bar{X}$ & $\mathrm{X}$ & \\
\hline Ophthalmological problems & $\bar{X}$ & $\mathrm{X}$ & \\
\hline Low blood pressure & $\bar{X}$ & & \\
\hline Blood-pressure drops & $\mathrm{X}$ & & \\
\hline
\end{tabular}




\section{Measures}

\section{Measures of Study 1}

We showed animated simulations of visual snow and of a blue field entoptic phenomenon for 10 seconds. The simulations were taken from VisionSimulations.com. Participants were instructed to pay attention to the dots of light and to replay the video as often as they wanted. They were then asked how frequently they see similar dots of light occupying their entire visual field with their eyes open in everyday life and instructed to rate the frequency on a visual analogue scale by positioning a cursor on a line anchored by $0 \%$ (Never) and $100 \%$ (All the time). The line had 101 possible positions and responses were rounded to fit percentages from $0 \%$ to $100 \%$ in 11 steps $(0 \%, 10 \%, 20 \%, \ldots, 100 \%)$. The simulation of visual snow can be found at https://visionsimulations.com/visual-snow.htm?background=restaurant.jpg. The simulation of the blue field entoptic phenomenon can be found at https $/ /$ visionsimulations.com/blue-fieldphenomena.htm.

Participants were also asked to report how frequently they see the dots of light representing visual snow when their eyes are closed using the same visual analogue scale.

Participants were shown a graphic representation of floaters (also known as muscae volitantes) and instructed to look at the appearing floater forms. They were then asked to rate how frequently they see similar forms in daily life using the same visual analogue scale.

The degrees of distress and fascination caused by visual snow were measured on a 7point Likert scale ranging from 1 (absolutely nothing) to 7 (extremely).

Two open-ended questions were included asking about situations that commonly trigger the appearance of visual snow and if there was a life event that could be associated with the first- 
time appearance of visual snow. If such triggering situations and life events existed, respondents were asked to describe them in their own words.

Participants were requested to tick options indicating analogies for their experience of visual snow (they could tick more than one option). Options were: a) static of an analog television, b) snowfall, c) uncertain, d) other analogies. If they chose "other analogies", they were asked to describe them.

We inquired since when visual snow had been seen. Options of answers were: a) since childhood, b) since some years ago, and c) since very recently. Answers b and c were collapsed into a category "since after childhood".

We asked about the intensification of visual snow with time. Options of answers were: a) the intensity has been increasing with time, b) the intensity has been decreasing with time, and c) the intensity has remained the same.

The computer-screen time was assessed by asking participants to estimate the number of hours per day they spend looking at a computer screen.

The frequency of tinnitus was assessed by asking participants about the amount of time they hear a ringing, buzzing, hissing, or other sounds that do not come from the external world. Answers were given on the same scales used to assess visual snow frequency, i. e., the frequency of experienced tinnitus was reported on a scale from $0 \%$ (Never) to $100 \%$ (All the time) with intervals of 10 .

The levels of distress and fascination caused by tinnitus were measured on a 7-point Likert scale from 1 (absolutely nothing) to 7 (extremely).

Participants were asked if they have migraine, ophthalmological problems, low blood pressure, and episodes of blood pressure drops. 
The propensity for attentional absorption was measured with the Modified Tellegen Absorption Scale (MODTAS) [26], which is composed of 34 self-descriptive items to be rated on a 5-point scale from 1 (I totally disagree) to 5 (I totally agree), which are assigned to the following five subscales [26]:

1) Imaginative Involvement: propensity to be attentionally absorbed in imagination. Examples of items: "If I wish I can imagine (or daydream) some things so vividly that they hold my attention as a good movie or story does."; "I can sometimes recollect certain past experiences in my life with such clarity and vividness that is like living them again or almost so".

2) Esthetic Involvement in Nature: propensity to be attentionally absorbed in external events. Examples of items: "It is sometimes possible for me to be completely immersed in nature or in art and feel as if my whole state of consciousness has somehow been temporarily altered"; "When I listen to organ or other powerful music, I sometimes feel as if I am being lifted into the air".

3) Altered States of Consciousness: tendency to experience psychological states that deviate markedly from the usual waking consciousness. Examples of items: "I think I really know what some people mean when they talk about mystical experiences"; "Sometimes I experience things as if they were doubly real'.

4) Synesthesias: proneness to experience associative synesthesias [38], i. e., when sensations in one particular sensory modality elicit mental associations in a different sensory modality, normally visual. Examples of items: "I find that different odors have different colors"; "Some music reminds me of pictures or changing color patterns".

5) Extrasensory Perception (ESP): tendency to be impressed by events that are intriguing because they apparently defy known physical laws. Examples of items: "I can often somehow 
sense the presence of another person before I actually see her/him"; "I often know what someone is going to say before he or she says it".

\section{Measures of Study 2}

Visual snow was assessed by asking participants to report the amount of time their visual field is full of tiny dots of light when their eyes are open (here we did not use visual simulations). Answers were given on an 11-point scale with options $0 \%$ (Never), 10\%, 20\%, . 100\% (All the time). We repeated the same question with the same scale to assess the frequency of visual snow with the eyes closed. We described visual snow as "dots of light" because that description is what is actually seen in most graphic simulations. As we will show in the Results section, not all people associate their experience of visual snow with static of analog television.

Measures similar to those in Study 1 were used to assess absorption, tinnitus frequency, tinnitus distress, tinnitus fascination, situations triggering visual snow, life events associated with the first appearance of visual snow, analogies of visual snow, intensification of visual snow over time, time of first-time appearance of visual snow, migraine, ophthalmological problems, and computer-screen time.

\section{Measures of Study 3}

Visual snow frequency was assessed as the amount of time the world was seen with many dots of light. Answers were given on an 11-point scale with options $0 \%$ (Never), 10\%, 20\%, .. $100 \%$ (All the time).

Absorption was measured like in Studies 1 and 2. 


\section{Results and discussion}

Prevalence of visual snow

Sample characteristics and the prevalence of visual snow across studies are reported in Table 2 and graphically depicted in Figure 1. Information regarding other variables of interest can be found in the Appendix.

Table 2. Sample characteristics and frequency of visual snow.

\begin{tabular}{|c|c|c|c|}
\hline & Study $1^{\mathrm{a}}$ & Study $2^{b}$ & Study $3^{\mathrm{c}}$ \\
\hline$N$ & 564 & 310 & 368 \\
\hline Women & 332 & 240 & 250 \\
\hline Men & 232 & 70 & 118 \\
\hline \multicolumn{4}{|l|}{ Age } \\
\hline Mean $(S D)$ & $32.43(16.68)$ & $30.77(11.57)$ & $27.18(8.53)$ \\
\hline Range & $18-75$ & $18-67$ & $18-75$ \\
\hline Interquartile range & 18 & 17 & 6 \\
\hline Measure of visual snow & $\begin{array}{l}\% \text { of time the visual } \\
\text { field looks like an } \\
\text { animated simulation } \\
\text { of visual snow }\end{array}$ & $\begin{array}{l}\% \text { of time the } \\
\text { visual field is full } \\
\text { of tiny dots of light }\end{array}$ & $\begin{array}{l}\% \text { of time the } \\
\text { world is seen with } \\
\text { many dots of light }\end{array}$ \\
\hline \multicolumn{4}{|l|}{$\begin{array}{l}\text { Estimated percentage of time } \\
\text { seeing visual snow }\end{array}$} \\
\hline $0 \%$ & 55.9 & 54.5 & 56.0 \\
\hline $10 \%$ & 7.6 & 9.0 & 15.2 \\
\hline $20 \%$ & 4.3 & 6.8 & 6.3 \\
\hline $30 \%$ & 3.9 & 5.2 & 6.5 \\
\hline $40 \%$ & 2.0 & 2.3 & 3.5 \\
\hline $50 \%$ & 3.7 & 6.5 & 3.8 \\
\hline $60 \%$ & 1.6 & 5.2 & 2.7 \\
\hline
\end{tabular}




\begin{tabular}{|lccc|}
\hline $70 \%$ & 1.4 & 4.2 & 2.4 \\
$80 \%$ & 3.2 & 2.3 & 1.4 \\
$90 \%$ & 2.1 & 1.6 & .8 \\
$100 \%$ & 14.4 & 2.6 & \\
\hline Percentage of time seeing & & & \\
visual snow with closed eyes & & 29.7 & \\
$0 \%$ & 30.2 & 11.7 & \\
$10 \%$ & 13.4 & 5.9 & \\
$20 \%$ & 12.0 & 9.7 & \\
$30 \%$ & 8.7 & 7.6 & \\
$40 \%$ & 6.8 & 10.3 & \\
$50 \%$ & 9.1 & 5.9 & \\
$60 \%$ & 5.2 & 4.8 & \\
$70 \%$ & 5.2 & 5.2 & \\
$80 \%$ & 3.1 & 3.4 & \\
$90 \%$ & 3.1 & 5.9 & \\
$100 \%$ & 3.1 & 5.1 & \\
\hline Visual snow present since & & & \\
childhood (subsample of those & 47.4 & & \\
with visual snow) & & & \\
\hline Progression of visual snow & & & \\
(subsample of those with visual & & & \\
snow) & & & \\
It has been increasing with time & & & \\
It has been decreasing with time & 12.2 & & \\
It has remained constant & 75.1 & & \\
\hline
\end{tabular}

${ }^{a}$ collected in 2020-2021; ${ }^{b}$ collected in 2018-2019; ${ }^{c}$ collected in 2017

${ }^{1}$ In the group of those not seeing visual snow, a small proportion $(\mathrm{N}=13 ; 2.4 \%$ of the total sample in Study 1) reported seeing it very occasionally. 


\section{Insert Figure 1. Frequency of visual snow}

The prevalence of those experiencing visual snow was remarkably similar regardless of whether the assessment was performed with the aid of animated graphic simulations or participants were simply asked about the frequency of seeing "dots of light". Roughly $45 \%$ of the respondents reported some visual experience of snow with varying degrees of frequency. In Study 1, 14\% [95\% CI: $11 \%$ - 17\%] of the respondents reported seeing visual snow permanently (100\% of the time). The percentage of those who always or nearly always see visual dots (between $80 \%$ and $100 \%$ of the time) was about $20 \%$ (95\% CI: $16 \%-23 \%$ ). Study 1 used animated graphic simulations of visual snow. When participants were asked about the frequency of seeing visual snow without the aid of graphic simulations, the proportion of those reporting seeing "dots of light" always or nearly always (between $80 \%$ and $100 \%$ of the time) was lower: 6.5\% (95\% CI: $4 \%-10 \%$ ) in Study 2 and 3.6\% (95\% CI: $2 \%-6 \%$ ) in Study 3. Visual snow may be a rather common phenomenon, but some people only notice it when instructed to pay attention to it, and the graphic simulation may have been more effective in calling attention to the fact that visual snow is "permanently or usually there". A similar thing happens with entoptic phenomena, which may only become visible after attention has been called to them. The use of graphic simulations is likely a more reliable method because it does not depend on descriptions of particular analogies. Therefore, it is possible that the results of Studies 2 and 3 are underestimations.

In a study with a representative British sample, the prevalence of visual snow occurring always or nearly always was estimated at 3.7\% (CI: 2.7-5.2) [18], which is similar to the results obtained in Studies 2 and 3. The British study did not use a graphic representation, but simply 
asked, 'Do you have visual symptoms that can be described like this: 'tiny dots, continuously occurring in the entire visual field? The dots are usually black/grey on white background or grey/white on a black background, but they can also be transparent, white flashing or colored" [18]. As noted above, when graphic simulations are not employed, many respondents might not associate their experience with the verbal descriptions offered. As shown in Table 3, only $67 \%$ of the respondents in Study 1 who experience visual snow reported that it resembled the static of analog television, which is the analogy most commonly given. One third of the sample did not make the association with static. One quarter was uncertain about what analogy to use (see Table $3)$.

Table 3. Analogies of visual snow (Study 1; $\mathrm{N}=209$ ).

\begin{tabular}{|ll|}
\hline Static of an analog television & $67 \%^{1}$ \\
Snowfall & $7.7 \%^{1}$ \\
Uncertain & $24.9 \%^{1}$ \\
Other analogies & $6.2 \%^{1}$ \\
Description of the other analogies & $.2^{2}$ \\
Grain in photos (not static from analog & $.2^{2}$ \\
television) & \\
Dark moving dots (in addition to luminous \\
static) \\
Non-luminous static with moving spots (in \\
addition to luminous static) \\
Out-of-focus image
\end{tabular}




\begin{tabular}{|ll|}
\hline Tiny colorful dots & $.2^{2}$ \\
Dust particles & $.2^{2}$ \\
Rounded clouds & $.2^{2}$ \\
As if seeing the wind & $.2^{2}$ \\
Fireflies & $.2^{2}$ \\
Falling purpurins & $.2^{2}$ \\
Sparks of the reflection of sunlight on water & $.2^{2}$ \\
As if cells falling from heaven & $.2^{2}$ \\
Angels & $.2^{2,3}$ \\
\hline
\end{tabular}

${ }^{1}$ Sum of percentages is more than $100 \%$ because some respondents ticked more than one option.

${ }^{2}$ Percentages of .2 refer to one single participant for each description.

${ }^{3}$ This respondent reported that visual snow appeared during a process of religious

transformation. This is more accurately described as a subjective interpretation than an analogy.

The results of the present study must be interpreted with caution. Our samples were not representative of the Portuguese population, although in comparison with other studies, the total number of respondents was large $(N=1,242)$. Future studies with more representative samples and stratified according to gender, education, and age are needed. The results still suggest a higher prevalence of visual snow in the general population than is often assumed and also indicate that visual snow is not an all-or-nothing phenomenon, i. e., it is not permanently present in the visual field of those who experience it. Visual snow appears to be more frequently seen with closed eyes. In Studies 1 and 2, around 70\% reported seeing visual snow with closed eyes (see Table 2 and Figure 1). 


\section{Visual snow and tinnitus}

In clinical groups, tinnitus is a consistent correlate of visual snow, and the two conditions may share common etiologies. This was replicated in the nonclinical groups in the present investigation. Table 4 shows a significant positive correlation between visual snow frequency and tinnitus frequency in Studies 1 and 2. There exists a wide variability in the frequency of both tinnitus and visual snow (see Figure 2), but tinnitus was experienced by a larger proportion of respondents than visual snow.

Table 4. Correlates of visual snow (Pearson correlation coefficients).

\begin{tabular}{|c|c|c|c|}
\hline & Study 1 & Study 2 & Study 3 \\
\hline Sex $($ women $=1$, men $=2)$ & .08 & -.05 & $.14^{*}$ \\
\hline Age & .01 & .01 & .09 \\
\hline Migraine & $.11 *$ & $.13 *$ & \\
\hline Tinnitus & $.23 * * *$ & $.37 * * *$ & \\
\hline Blue-field entoptic phenomenon & $.39 * * *$ & & \\
\hline Floaters & $.21 * * *$ & & \\
\hline Visual snow since childhood ${ }^{1}$ & .02 & -.06 & \\
\hline Distress caused by visual snow & $.37 * * *$ & .03 & \\
\hline Fascination with visual snow & $.18^{*}$ & $.32 * * *$ & \\
\hline Absorption (MODTAS total score $^{2}$ ) & $.17 * * *$ & $.21 * * *$ & $.34 * * *$ \\
\hline MODTAS Imaginative involvement ${ }^{2}$ & $.13 * *$ & $.18 * *$ & $.28 * * *$ \\
\hline MODTAS Esthetic involvement in & $.11 *$ & $.16^{* *}$ & $.26 * * *$ \\
\hline
\end{tabular}




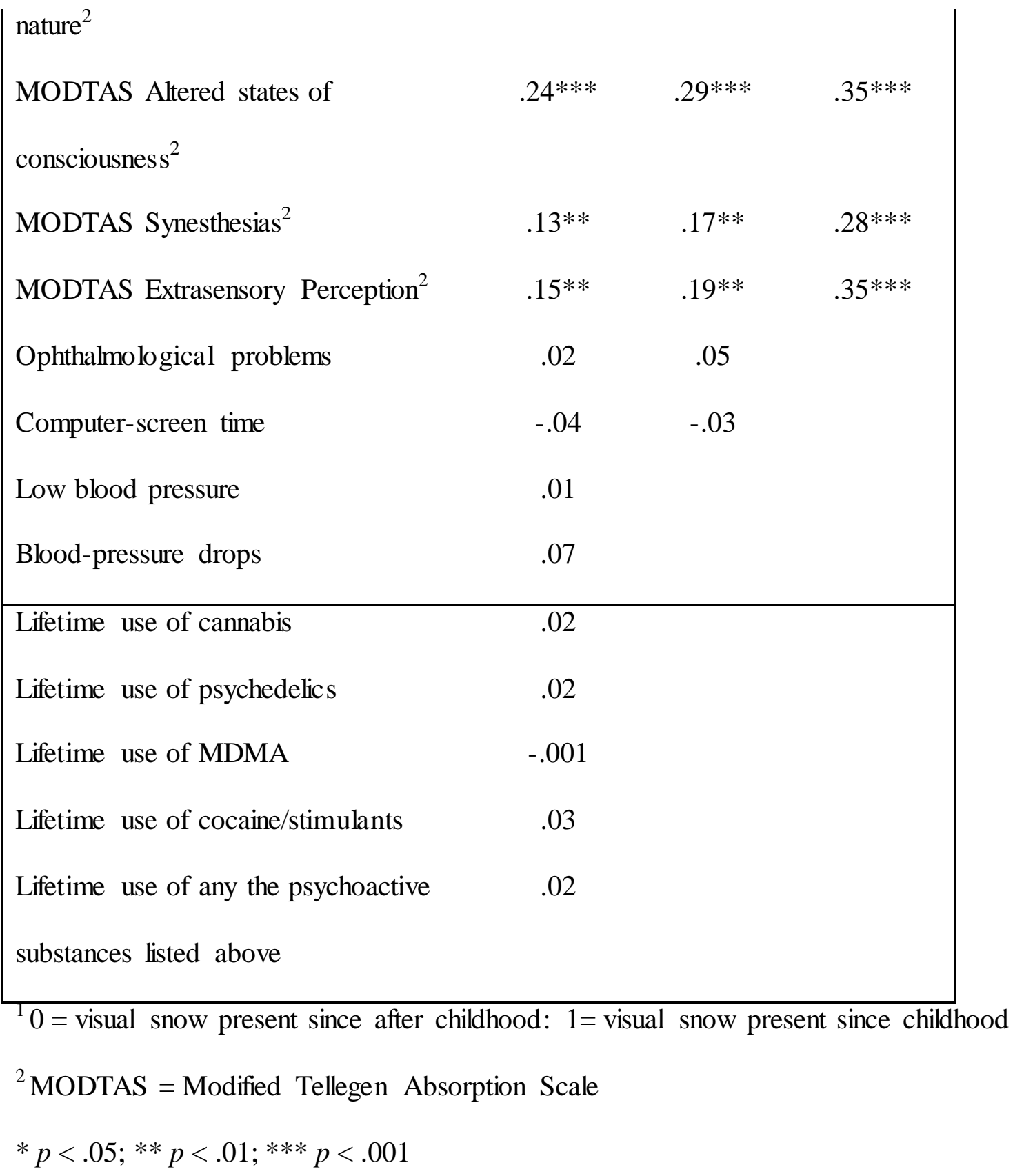

\section{Insert Figure 2. Frequency of tinnitus}

\section{Reactions to visual snow and tinnitus}

The emotional responses (distress and fascination) provoked by visual snow were quite similar regardless of whether visual snow had been assessed with the aid of animated graphic 
simulations or not. In Studies 1 and 2, the levels of distress resulting from visual snow varied considerably (see Figure 3), but most respondents (between 63\% and 69\%) do not feel distressed, and if they do, the distress is minimal. Between $30 \%$ and $32 \%$ of those seeing visual snow reported absolutely no distress (scoring 1 point on the 7 -point distress scale). Between $37 \%$ and $38 \%$ reported minimal or little distress (scoring 2 and 3 points). Between $10 \%$ and $19 \%$ reported moderate distress (scoring 4 points). Between $18 \%$ and $20 \%$ complained of higher levels of distress (scoring between 5 and 7 points). Although many people are not distressed by their visual snow, there appears to be a substantial proportion that could possibly benefit from clinical attention. As with tinnitus, pharmacologic interventions for visual snow are largely ineffective. Possible strategies to help people who are distressed by visual snow are cognitive-behavioral therapies like those used for tinnitus distress. The objective would not be to eliminate visual snow, but to eliminate the associated distress [39].

\section{Insert Figure 3. Distress and fascination with visual snow.}

The distribution of distress caused by visual snow is similar to that of tinnitus (see Figure 4). Most respondents with tinnitus were not distressed at all, and if they were, distress was minor. Between $22 \%$ and $30 \%$ reported higher levels of distress with tinnitus (scoring between 5 and 7 points on the 7-point distress scale). It is well known that many people are not distressed by tinnitus $[40,41]$.

\section{Insert Figure 4. Distress and fascination with tinnitus}


Because sometimes unusual visual phenomena cause fascination, we investigated whether visual snow also evokes this positive reaction. Some participants reported higher degrees of fascination with visual snow; 9\% in Study 1 and 24\% in Study 2 scored between 5 and 7 on the 7-point fascination scale (see Figure 3). Fewer people reported fascination with tinnitus (see Figure 4).

\section{Visual snow and entoptic phenomena}

Study 1 demonstrated that visual snow frequency correlated directly with the frequency of two entoptic phenomena: floaters and blue field entoptic phenomenon (see Table 4). This coincides with research in clinical samples in which visual snow and entoptic phenomena often occur together. Entoptic phenomena are among the additional visual phenomena required to diagnose the visual snow syndrome [1-3]. More people apparently report visual snow with additional visual phenomena than without $[3,18]$.

\section{Visual snow and migraine}

Table 4 shows weak but significant associations between migraine and visual snow frequency, whether visual snow was measured with animated graphic simulations or by mere verbal description. This coincides with studies in clinical samples showing that migraine is a correlate of visual snow. The study with a British representative sample [18] demonstrated a marginal association between the visual snow syndrome and migraine, which disappeared in a multiple regression in which tinnitus was the only significant predictor. The question whether visual snow is more strongly associated with migraine in clinical groups deserves further investigation. 


\section{Triggers of visual snow}

Because many people who see visual snow do not see it all the time, it is important to ascertain if there are situations that trigger short-term appearances of visual snow. Only some respondents with visual snow reported such triggers (31\% in Study 1 and $26 \%$ in Study 2 among those seeing visual snow). As shown in Tables 5 and 6, we detected eight types of triggers: lightrelated, attention-related, tiredness-related, blood pressure-related, mood-related, eye-related, migraine-related, and pain-related. For those reporting light-related triggers, visual snow appears when looking at intense lights, when changing from dark to bright environments or when being in dark surroundings. Attention-related triggers refer to situations in which visual snow appears as a result of highly focused attention on something, but "vague thoughts" or "looking at the void" can also trigger visual snow, which indicates rather dispersed attention. Attention-related and light-related triggers can overlap, as visual snow can appear when focusing attention on lights. Visual snow can also appear when one is tired. Visual snow can become visible when drops in blood pressure are felt or as a consequence of movements that lower blood pressure. Mood-related triggers are more common with negative mood changes. Eye-related triggers are the result of a variety of physiological processes in the eyes, such as making pressure on the eyes or feeling "tired eyes". All these six types of triggers were equally observed in Studies 1 and 2, regardless of whether animated simulations of visual snow were presented or not (see Tables 5 and 6). Although migraine was related to visual snow in the present investigation, only two participants in Study 1 associated migraine attacks with the appearance of visual snow. Four additional participants in Study 1 reported visual snow triggered by pain (headache in one case, back pain in one, and unspecified in the two others). 
Table 5. Triggers of visual snow (Study $1 ; \mathrm{N}=78$ ): number of participants reporting particular types of triggers are indicated in parentheses (some participants reported more than one type) ${ }^{1}$.

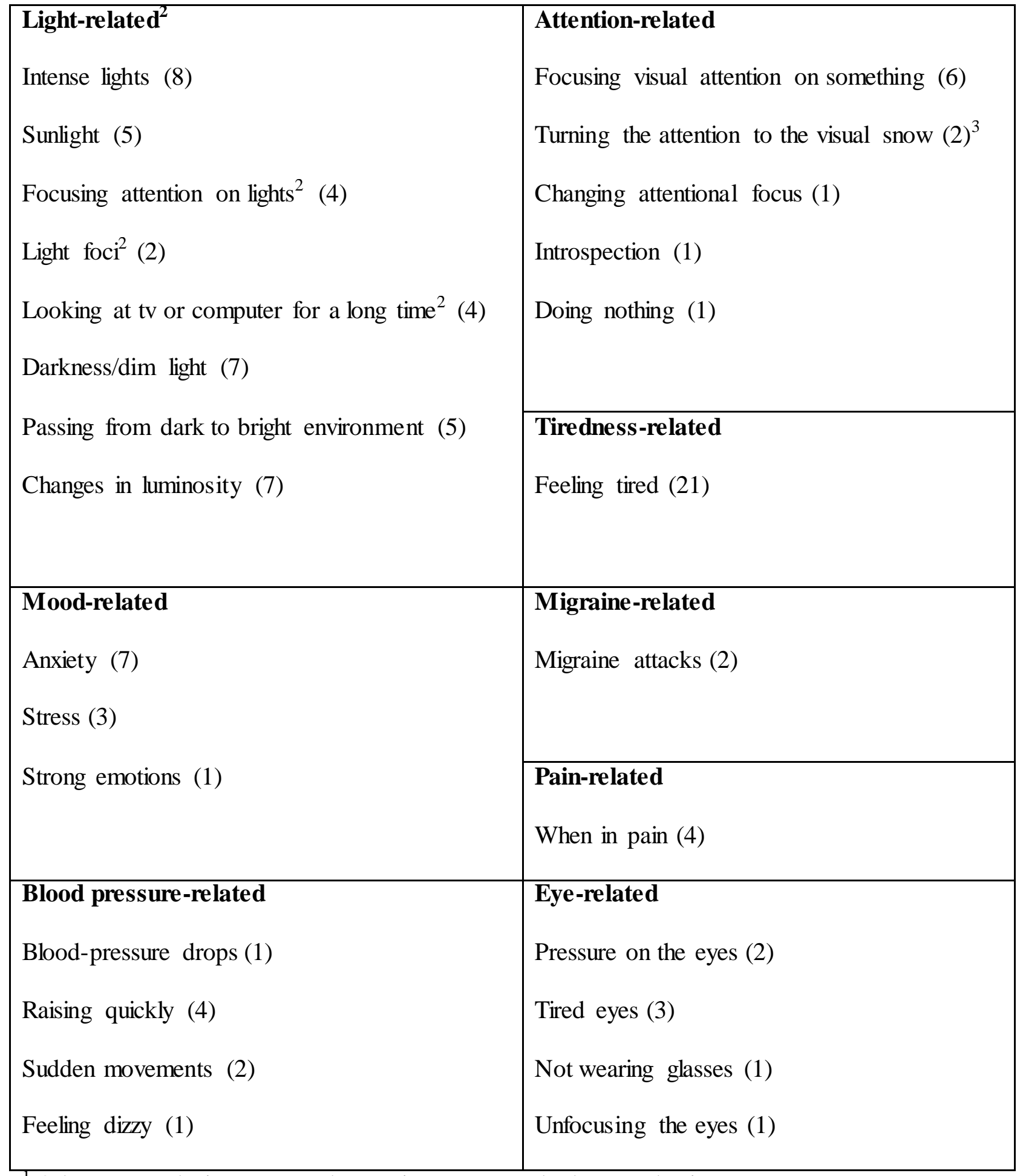

\footnotetext{
${ }^{1}$ Other reported triggers were hot environments and when awakening.
} 
${ }^{2}$ Some light-related triggers are also attention-related.

${ }^{3}$ One respondent reported that paying attention to visual snow is easier in nature.

Table 6. Triggers of visual snow (Study 2; $\mathrm{N}=35$ ): number of participants reporting particular types of triggers is indicated in parentheses (some participants reported more than one type).

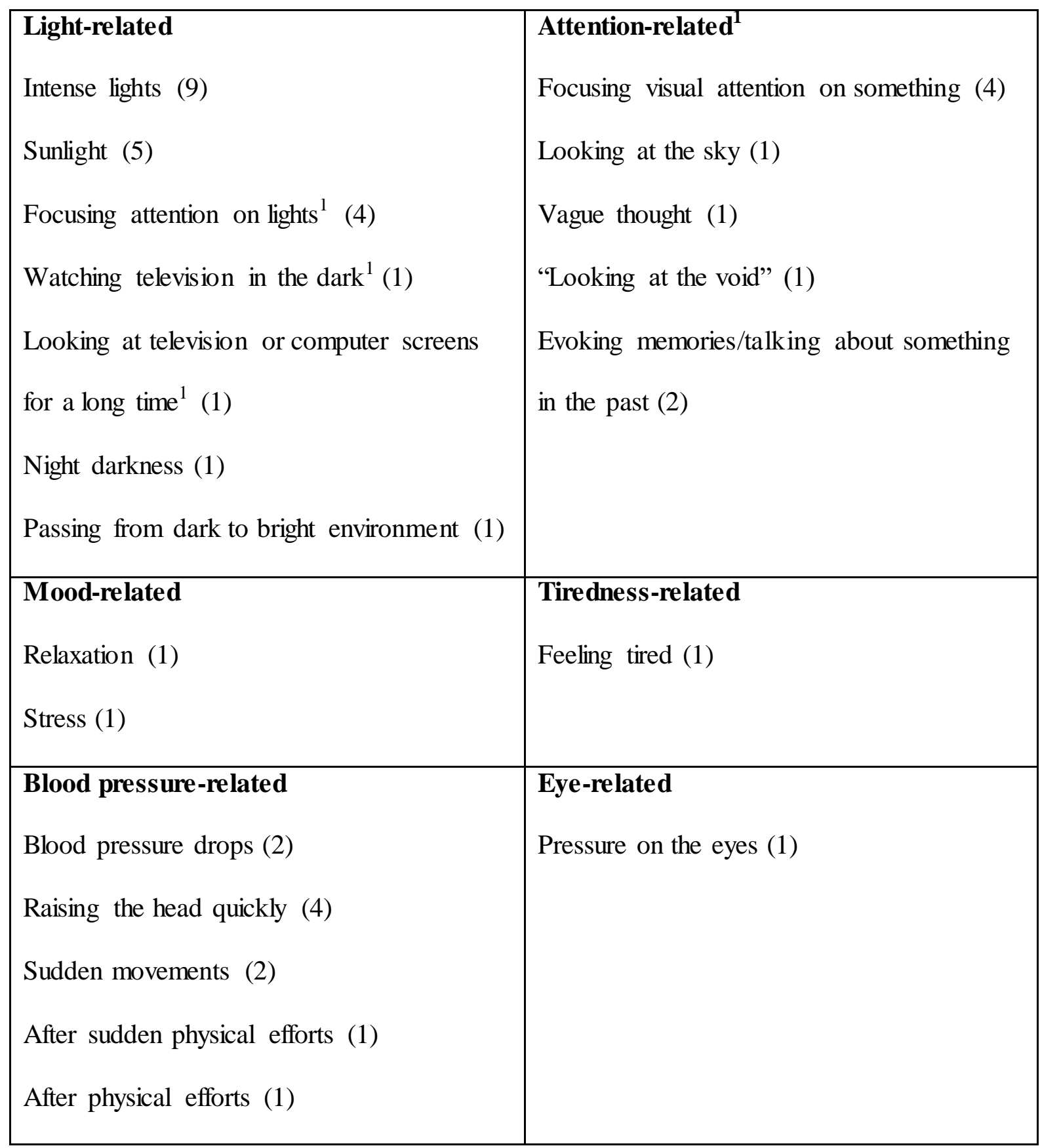


${ }^{1}$ Some attention-related triggers are also light-related.

Although reductions in blood pressure were a relatively common trigger, visual snow frequency was unrelated to self-reported low blood pressure and drops in blood pressure. Tiredness was a common trigger, especially in Study 1. Because fatigue has been associated with hypotension $[42,43]$, future research is needed to ascertain the interrelationships between visual snow and fatigue and low blood pressure.

Intense light was a common trigger, and some participants specifically indicated that looking at television or computer screens elicited visual snow. We tested whether computerscreen time correlated with visual snow frequency, but found no supportive evidence. In Studies 1 and 2, computer-screen time was uncorrelated with visual snow frequency (see Table 4).

We inquired about events that respondents associated with the first appearance of visual snow. Very few reported any such event, and the answers were very heterogeneous, as shown in Table 7. Some individuals may have reported non-causative, coincidental events. Only one respondent associated the use of substances (cannabis and synthetic cannabinoids) with the firsttime appearance of visual snow. Table 4 indicates that lifetime use of a variety of psychoactive substances was unrelated to visual snow frequency. We also found visual snow to be unrelated to ophthalmological problems (see Table 5), which is consistent with the extant research. Since three participants associated the first appearance of visual snow with ophthalmological problems, some etiologies of visual snow might be related to eye disorders.

Table 7. Events associated with the first appearance of visual snow: numbers of participants reporting a particular event are indicated in parentheses. 
\begin{tabular}{l} 
Study $\mathbf{1}$ (N = 12) \\
Ophthalmological problems (3) \\
Migraine (2) \\
Panic attacks (4) \\
'Waking in the direction of the sunset, as a child" (1) \\
Watching the sea (1) \\
Religious conversion to Christ (1) 1 \\
\hline Study 2 (N = 6) \\
Use of cannabis and synthetic cannabinoids (1) \\
Intensive practice of sports (1) \\
Loss of family member (1) \\
Change to vegan diet (1) \\
During a hospital stay in childhood, when walking at night in the hospital corridors (1) \\
In childhood, dots of light appeared associated with fear of dark, and were imagined to be \\
witches. They disappeared and reappeared later during university classes when attention was \\
very focused on teachers to the point that it was like surrounding space did not exist (1)
\end{tabular}

${ }^{1}$ This respondent associated visual snow with angels as an analogy (see Table 5).

The finding that very few participants could remember any event associated with the origin of visual snow corresponds with the study by Puledda and colleagues, in which $40 \%$ $46 \%$ reported seeing visual snow since childhood (for as long as they could recall). The majority of those in whom it started after childhood could not recall any associated event [3]. The associated events, when recalled, varied [3]. Many participants in Study 1 (47\%) and Study 2 
(51\%) reported that they have visual snow since childhood, as depicted in Table 2. These figures were similar to those obtained by Puledda and colleagues. Table 4 shows that visual snow frequency was uncorrelated with seeing visual snow since childhood.

Table 2 demonstrates that visual snow intensity usually remains stable. In a study by Schankin and colleagues, $41 \%$ of participants reported that their visual snow intensity had been stable from the start [2]. Among those with an initial phase of progressive intensification, $48 \%$ reported a later stabilization. Among those with a stepwise beginning, 100\% reported that stabilization followed [2].

\section{Visual snow and absorption}

Table 7 shows that the frequency of visual snow correlated positively with trait absorption, as well as with all the five dimensions: imaginary involvement, synesthesias, sensory involvement, extrasensory perception, and altered states of consciousness. This was found in all three studies, regardless of how visual snow was assessed. More frequent visual snow was associated with a greater capacity to be engrossed in sensory and imaginary experiences that are perceived as fascinating. The correlation with the dimension "altered states of consciousness" was always the strongest in the three studies, raising the possibility that people with visual snow might be more susceptible to hypnosis [24-26,32,34,36], more open to enrapturing esthetic experiences, feel the effects of psychoactive substances more intensely, have more blissful sexual experiences [44], and be more prone to mystical-type experiences [25,37], which have recently become a topic of growing scientific interest [25,37,45-49]. These are important issues for future research, especially because some altered states of consciousness can be very enriching [48]. 
A possible explanation for the relationship between absorption and visual snow is that visual cortex hyperexcitability facilitates visual snow and intensifies perceptual and imaginary experiences to the extent that they absorb attention to a high degree. Possible brain areas that may be involved in visual snow experiences are the associative visual cortex and the thalamus [5]. A possible next step could be to study the general excitability of the associative visual cortex and the activity of the thalamus in participants experiencing visual snow and showing a greater capacity to be engrossed in visual experiences and imagination. Visual receptors and neurons demonstrate continuous activity with or without sensory information on the retinae. Neural activity in visual areas without sensory stimulation is typically labeled visual noise. Such lowerlevel noise activity may be suppressed by certain brain areas, possibly including the thalamus, and not enter awareness. One possible explanation for visual snow experiences may be less suppression or lower consciousness thresholds in observers with hyperexcitability of the visual cortex.

An additional explanation for the link between visual snow and absorption is that both might be influenced by patterns of serotonergic activity, particularly at the $5 \mathrm{HT}_{2 \mathrm{a}}$ receptors [5052]. The serotonergic agonist lysergic acid diethylamide (LSD) increases visual cortex activity and induces the so-called ego dissolution [53], i. e., loss of the usual perception of the limits between the self and the external world, which is a characteristic of states of absorbed attention [24]. The psychoactive effects of LSD and psilocybin are thought to be largely mediated by agonist activity on the $5 \mathrm{HT}_{2 \mathrm{a}}$ receptors [53].

\section{Final remarks}


Visual snow seems to be a relatively common phenomenon with many people experiencing it always or nearly always. Many people are not distressed, implying that it is not a distressing phenomenon per se. In the nonclinical group in the present investigation, we found visual snow correlates similar to those that have already been observed in clinical groups: tinnitus, entoptic phenomena, and migraine. We also confirmed that visual snow is associated with a greater capacity to be attentionally absorbed, i. e., the capacity to be fascinated.

Could clinical cases be characterized by more intense visual snow? It is possible, but difficult to confirm. Visual snow is an inherently subjective experience. An intensity that is comfortable for one person could be excessive and distressful for another. If one is anxious about the experience of visual snow or desires to conform to what is perceived to be "normal visual perception", this person might seek clinical advice. These aspects deserve to be addressed by clinicians. Cognitive-behavioral therapies might offer a possible intervention to reduce distress related to visual snow. In some cases, reassuring distressed people that visual snow can be a normal experience may already be an effective intervention.

There exist anecdotal reports of people who talked to their parents about the visual snow when they were children and were shocked by their parents' lack of understanding (e. g. [54]). Parental education in this regard seems important. As shown in Table 7, one respondent interpreted visual snow as terrifying witches when she was child. It is important to raise attention to visual snow in pediatric care.

An important question still needs be asked. If there are so many people who are not distressed and even enjoy visual snow, why has scientific attention been so focused on people who are distressed? A possible answer lies in the difficulties that many people have relating their subjective experiences, fearing being misunderstood. An example is provided by a study of 
women with visual synesthesias during sexual activity, i. e, women who see colorful forms while experiencing sexual desire, arousal, or orgasm. These experiences are pleasant and enriching and are associated with greater sensory and emotional involvement (absorption) in sexual intercourse. Nevertheless, many of these women felt dissatisfied because they had difficulties sharing their synesthetic experiences with their partners [55]. Perhaps it is time to be more open and accepting of these possibly fascinating and intriguing aspects of human experience. Increasing awareness of visual snow by talking about it is one important step. To extend understanding to many other intriguing phenomena in visual or other sensory modalities, experienced as pleasant or not, will be another step. Conducting research like ours could help foster awareness of these phenomena.

More research is needed to establish the prevalence of visual snow and its underlying neurophysiological determinants, to better understand its psychological and behavioral correlates, and to characterize in greater detail emotional reactions to it. 
There are no conflicts of interest. 


\section{References}

1. Puledda F, Schankin C, Digre K, Goadsby PJ. Visual snow syndrome: What we know so far. Curr Opin Neurol 2018; 31:52-58.

2. Schankin CJ, Maniyar FH, Digre KB, Goadsby PJ. 'Visual snow': a disorder distinct from persistent migraine aura. Brain 2014; 137:1419-1428.

3. Puledda F, Schankin C, Goadsby PJ. Visual snow syndrome: A clinical and phenotypical description of 1,100 cases. Neurology 2020; 94: e564-e574.

4. van Dongen RM, Waaijer LC, Onderwater GLJ, Ferrari MD, Terwindt GM. Treatment effects and comorbid diseases in 58 patients with visual snow. Neurology 2019; 93: e398e403.

5. Lauschke JL, Plant GT, Fraser CL. Visual snow: A thalamocortical dysrhythmia of the visual pathway? J Clin Neurosci 2016; 28:123-127.

6. Schankin CJ, Maniyar FH, Sprenger T, Chou DE, Eller M, Goadsby PJ. The relation between migraine, typical migraine aura and "visual snow." Headache 2014; 54: 957-966.

7. White OB, Clough M, McKendrick AM, Fielding J. Visual snow: visual misperception. J Neuro Ophthalmol 2018; 38:514-521.

8. Schankin CJ, Maniyar FH, Chou DE, Eller M, Sprenger T, Goadsby PJ. Structural and functional footprint of visual snow syndrome. Brain 2020; 143:1106-1113.

9. Fraser CL, White OB. There's something in the air. Surv Ophthalmol 2019; 64:729-733.

10. Puledda F, Ffytche D, Lythgoe DJ, O’Daly O, Schankin C, Williams SCR, Goadsby PJ. Insular and occipital changes in visual snow syndrome: a BOLD fMRI and MRS study. Ann Clin Transl Neurol 2020; 7:296-306. 
11. McKendrick AM, Chan YM, Tien M, Millist L, Clough M, Mack H et al. Behavioral measures of cortical hyperexcitability assessed in people who experience visual snow. Neurology 2017; 88:1243-1249.

12. Eren O, Rauschel V, Ruscheweyh R, Straube A, Schankin CJ. Evidence of dysfunction in the visual association cortex in visual snow syndrome. Ann Neurol 2018; 84:946-949.

13. Eren O, Schankin CJ. Insights into pathophysiology and treatment of visual snow: a systematic review. Prog Brain Res 2020; 255: 311-326.

14. Mehta DG, Garza I, Robertson CE. Two hundred and forty-eight cases of visual snow: A review of potential inciting events and contributing comorbidities. Cephalgia 2021;41: 10151026.

15. Aldusary N, Traber GL, Freund P, Fierz FC, Weber KP, Baeshen A, Alghmadi J, Saliju B, Pazahr S, Mazloum R, Alsheri F, Landau K, Kollias S, Piccirelli M, Michels L. Abnormal connectivity and brain structure in patients with visual snow. Front Hum Neurosci 2020; 14 : 582031.

16. Puledda F, Bruchhage M, O’Daly O, Ffytche D, Williams SCR, Goadsby PJ. Occipital cortex cerebellum gray matter changes in visual snow syndrome. Neurology 2020; 95: e1792e1799.

17. Liu GT, Schatz NJ, Galetta SL, Volpe NJ. Persistent positive visual phenomena in migraine. Neurology 1995; 45:664-668.

18. Kondziella D, Olsen MH, Dreier JP. Prevalence of visual snow syndrome in the UK. Eur J Neurol 2020; 27:764-772.

19. Wood H. Shedding new light on visual snow syndrome. Nat Rev Neurol 2020; 16: 183. 
20. Raghavan M, Remler BF, Rozman S, Pelli DG. Patients with visual "snow" have normal equivalent input noise levels. Invest Ophth Vis Sci 2010; 51: 1808.

21. Jung RE, Flores RA, Hunter D. A new measure of imagination ability: anatomical brain imaging correlates. Front Psychol 2016; 7:496.

22. Benedek M, Jauk E, Beaty R, Fink A, Koschutnig K, Neubauer AC . Brain mechanisms associated with internally directed attention and self-generated thought. Sci Rep 2016; 6:22959.

23. Leshikar ED., Duarte A., \& Hertzog C. Task-selective memory effects for successfully implemented encoding strategies. Plos One 2012; 7:e38160.

24. Tellegen A, \& Atkinson G. Openness to absorbing and self-altering experiences (“absorption"), a trait related to hypnotic susceptibility. J Abnorm Psychol 1974; 83:268277.

25. Lifschitz M, van Elk M, Luhrmann TM. Absorption and spiritual experience: a review of evidence and potential mechanisms. Conscious Cogn 2019; 73:102760.

26. Jamieson GA. The Modified Tellegen Absorption Scale: a clearer window on the structure and meaning of absorption. J Australian Clin Exp Hypn 2005; 33:119-139.

27. Tellegen A. Practicing the two disciplines for relaxation and enlightenment: Comment on "Role of the feedback signal in electromyography biofeedback: The relevance of attention" by Qualls and Sheehan. J Exp Psych 1981; 110:217-226.

28. Costa RM, Pestana J, Costa D. Self-transcendence, sexual desire, and sexual frequency. J Sex Marital Ther 2018; 44:56-60.

29. Glisky ML, Tataryn DJ, Tobias BA, Kihlstrom JF, McKonkey KM. Absorption, openness to experience, and hypnotizability. J Pers Soc Psychol 1991; 60: 263-272. 
30. Roche SM, McConkey KM. Absorption: nature, assessment, and correlates. J Pers Soc Psychol 1990; 59: 91-101.

31. Costa RM, Oliveira TF, Pestana J, Costa D. Self-transcendence is related to higher female sexual desire. Pers Indiv Dif 2016; 96: 191-197.

32. Radtke, H. L., \& Stam, H. J. (1991). The relationship between absorption, openness to experience, anhedonia, and susceptibility. Int J Clin Exp Hypn 1991; 39: 39-56.

33. Wild TC, Kuiken D, Schopflocher D. The role of absorption in experiential involvement. J Pers Soc Psychol 1995; 69: 569-579.

34. Crawford HJ. Hypnotizability, daydreaming styles, imagery vividness, and absorption: a multidimensional study. J Pers Soc Psychol 1982;45: 915-926.

35. Merckelback H, Horselenberg R, Muris P. The Creative Experiences Questionnaire (CEQ): a brief self-report measure of fantasy proneness. Pers Indiv Dif 2001; 31:987-995.

36. Hoyt IP, Nadon R, Register PA, Chorny J, Fleeson W, Grigorian EM, Otto L, Kihlstrom JF. 1989. Daydreaming, absorption and hypnotizability. Int J Clin Exp Hypn 1989; 37:332-342.

37. Cortes DS, Skragge M, Döllinger L, Laukka P, Fischer H, Nilsson ME, Hovey D, Westberg L, Larsson M, Granqvist P. Mixed support for a causal link between single dose intranasal oxytocin and spiritual experiences: opposing effects depending on individual proclivities for absorption. Soc Cogn Affect Neurosci 2018; 13: 921-932.

38. Neckar M, Bob P. Neuroscience of synesthesia and cross-modal associations. Rev Neurosci $2014 ; 25: 833-840$.

39. Marks E, Smith P, McKenna L. I wasn't at war with noise: How mindfulness based cognitive therapy changes patients' experiences of tinnitus. Front Psychol 2020; 11: 483. 
40. McCormack A, Edmonson-Jones M, Somerset E, Hall D. A systematic review of the reporting of tinnitus prevalence and severity. Hear Res 2016; 337: 70-79.

41. Vanneste S, Mohan A, De Rider D, To WT. The BDNF Val ${ }^{66}$ Met polymorphism regulates vulnerability to chronic stress and phantom perception. Prog Brain Res 2021; 260: 301-326.

42. Wessely S, Nickson J, Cox B. Symptoms of low blood pressure: a population study. BMJ 1990; 30: 362-365.

43. Pilgrim JA, Stansfeld S, Marmot M. Low blood pressure, low mood? BMJ 1992; 304: 75-78.

44. Ellero J, Costa RM. Altered states of consciousness, absorption, and sexual responsiveness. Psicol Saúde Doenças 2020; 21: 782-795.

45. Barrett FS, Griffiths RR (2018). Classical hallucinogens and mystical experiences: phenomenology and neural correlates. Curr Top Behav Neurosci 2018; 36: 393-430.

46. Taves A. Mystical and other alterations in the sense of self: an expanded framework for studying nonordinary experiences. Perspect Psychol Sci 2020; 15: 669-690.

47. Walach H. Inner experience - direct access to reality: a complementarist ontology and dual aspect monism support a broader epistemology. Front Psychol 2020; 11: 640.

48. Wittmann M. Altered states of consciousness: Experiences out of time and self. Cambridge, MA: MIT Press. 2020.

49. Cristofori I, Bulbulia J, Shaver JH, Wilson M, Krueger F, Grafman J. Neural correlates of mystical experience. Neuropsychologia 2016; 80: 212-220.

50. Eren OE, Schöberl F, Schankin CJ, Straube A. Visual snow after start of citalopram - novel insights into underlying pathophysiology. Eur J Clin Pharmacol 2021; 77: 271-272. 
51. Bachner-Melman R, Dina C, Zohar Ah, Constantini N, Lerer E, Hoch S, Sella S, Nemanov L. Gritsenko I, Lichtenberg P, Granot R, Ebstein RP. AVPR1a and SCL6A4 gene polymorphisms are associated with creative dance performance. PloS Genet 2005; 1:e42.

52. Ott U, Reuter M, Henning J, Vaitl D. Evidence for a common biological basis of the Absorption trait, hallucinogen effects, and positive symptoms: epistasis between 5-HT2a and COMT polymorphisms. Am J Med Genet B Neuropsychiatr Genet 2005; 137B: 29-32.

53. Carhart-Harris RL, Muthukumaraswamy S, Roseman L, Kaelen M, Droog W, Murphy K, Tagliazucchi E, Schenberg EE, Nest T, Orban C, Leech R, Williams LT, Williams TM, Bolstridge M, Sessa B, McGonigle J, Sereno MI, Nichols D, Hellyer PJ, Hobden P, Evans J, Singh KD, Wise RG, Curran HV, Feilding AF, Nutt DJ. Neural correlates of LSD experience revealed by multimodal neuroimaging. Proc Natl Acad Sci U S A 2016; 113: 4853-4858.

54. Grant A, Chung S. Transiet visual changes in children: positive and negative phenomena. Knights Templar Eye Foundation. Pediatric Ophthalmology Education Center 2015. https://www.aao.org/disease-review/transient-visual-changes- in-children-positive-ne ga

55. Nielsen J, Krüger THC, Hartmann U, Passie T, Fehr T, Zedler M. Synaesthesia and sexuality: the influence of synaesthetic perceptions on sexual experience. Front Psychol 2013; 4: 751. 

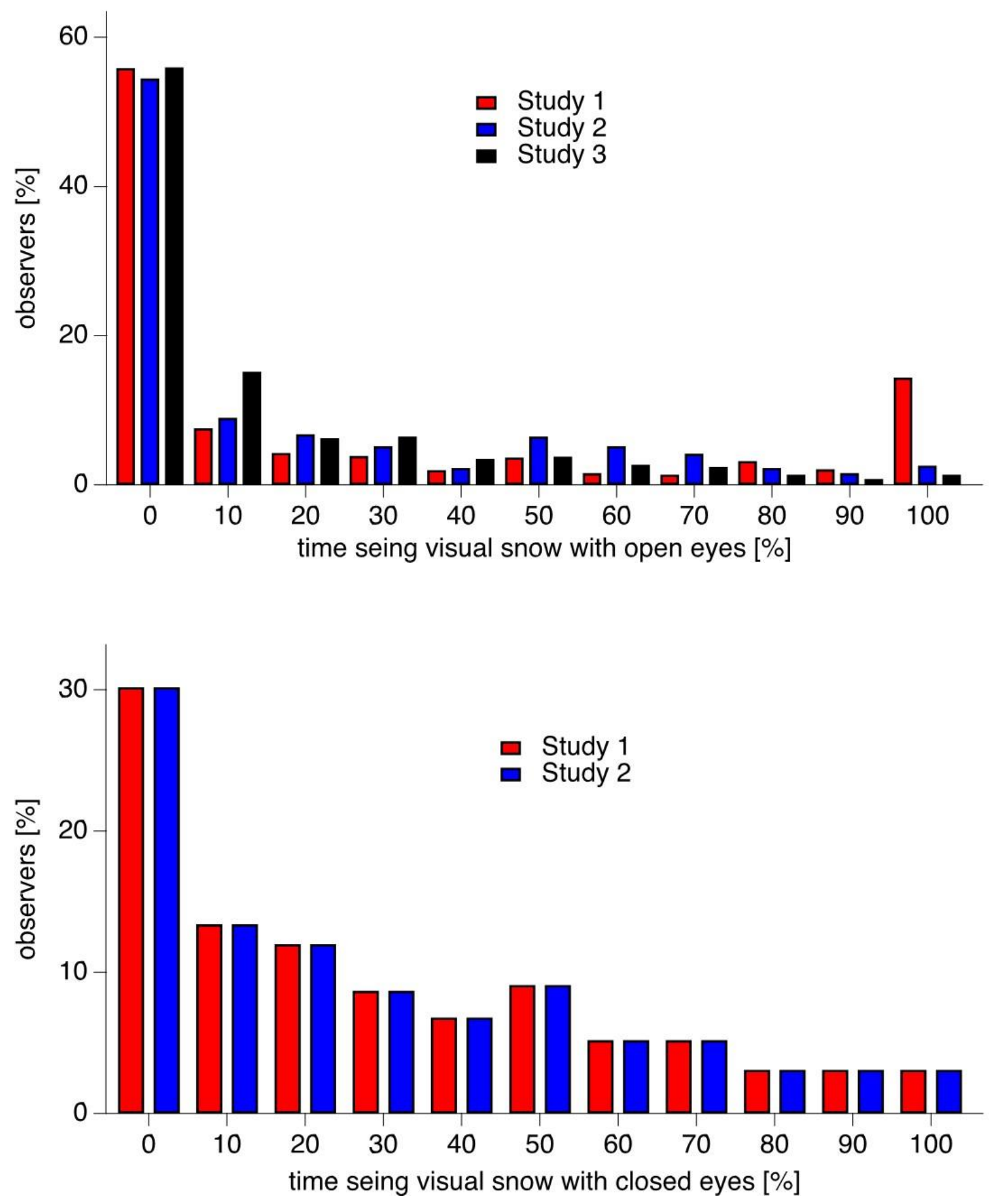

Figure 1. Frequency of visual snow. 


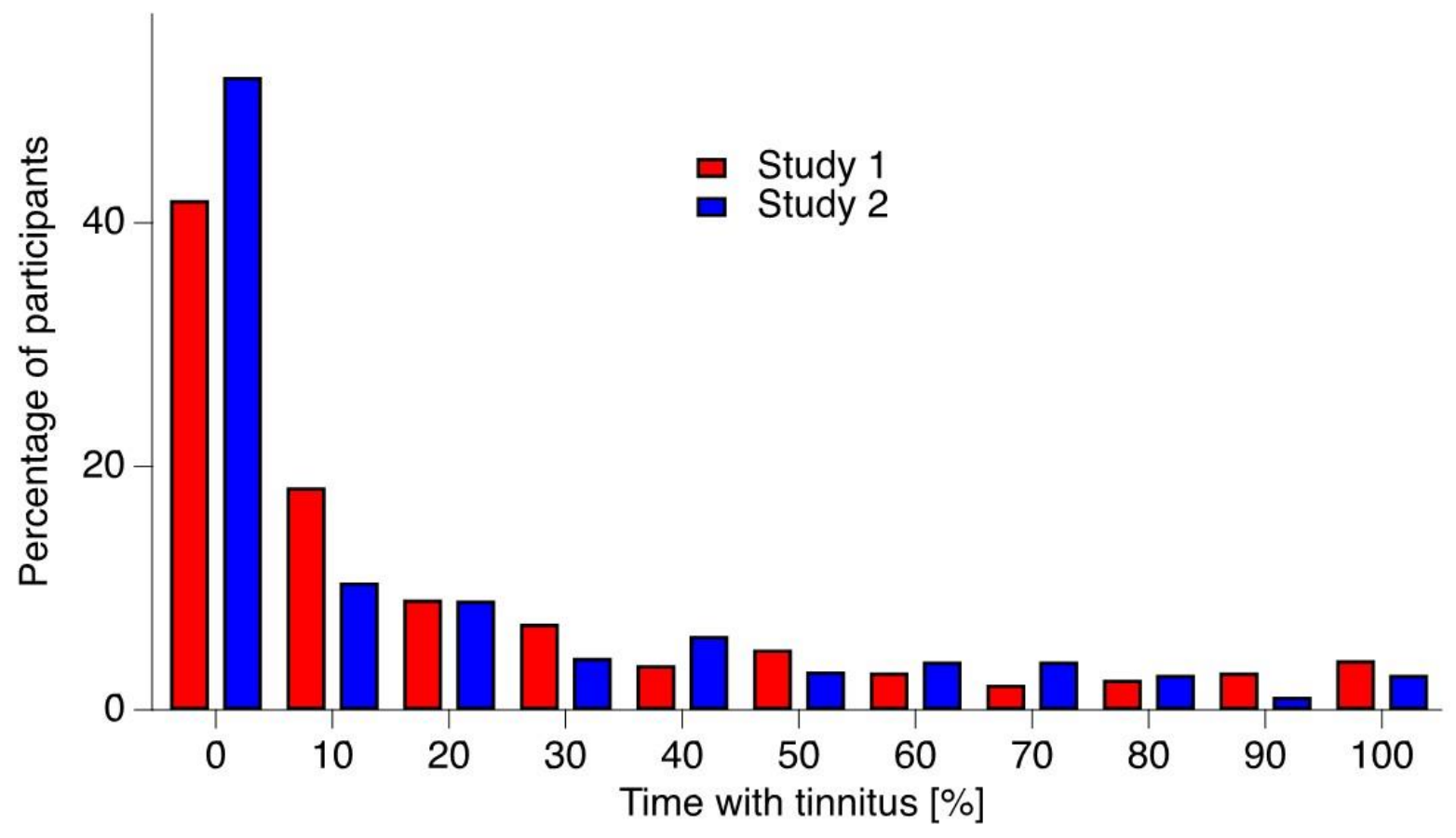

Figure 2. Frequency of tinnitus. 

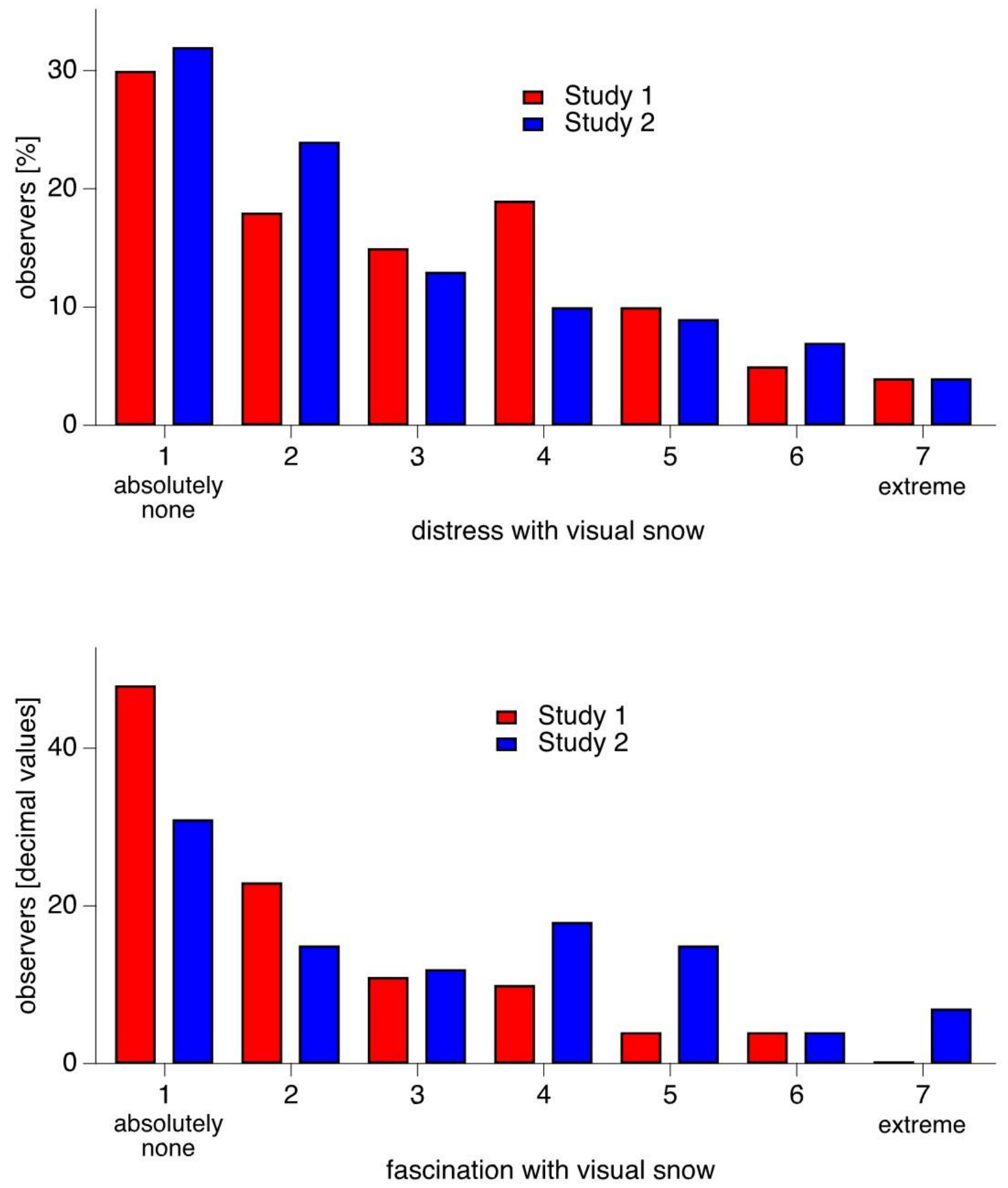

Figure 3. Distress and fascination with visual snow. 


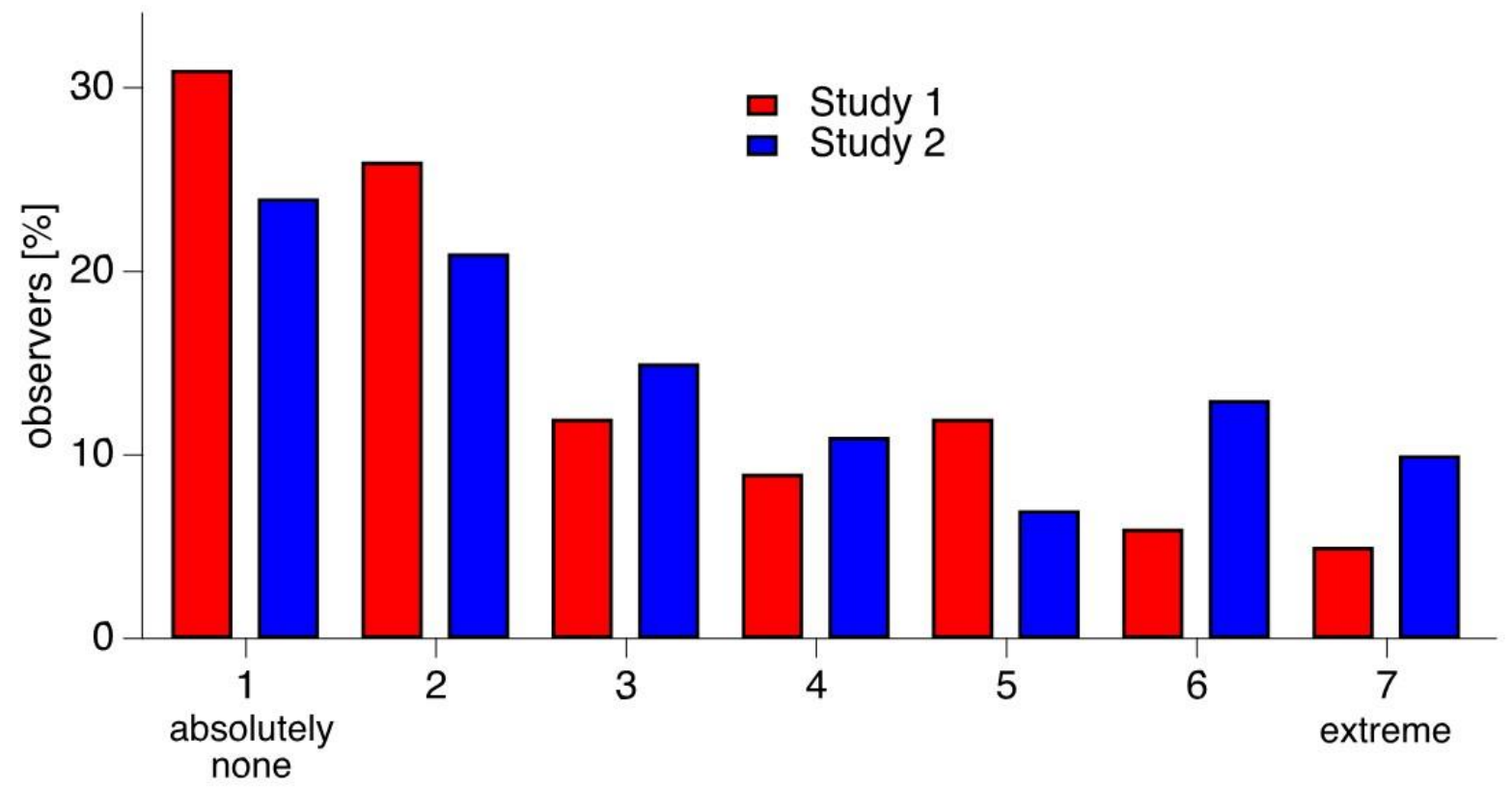

distress with tinnitus

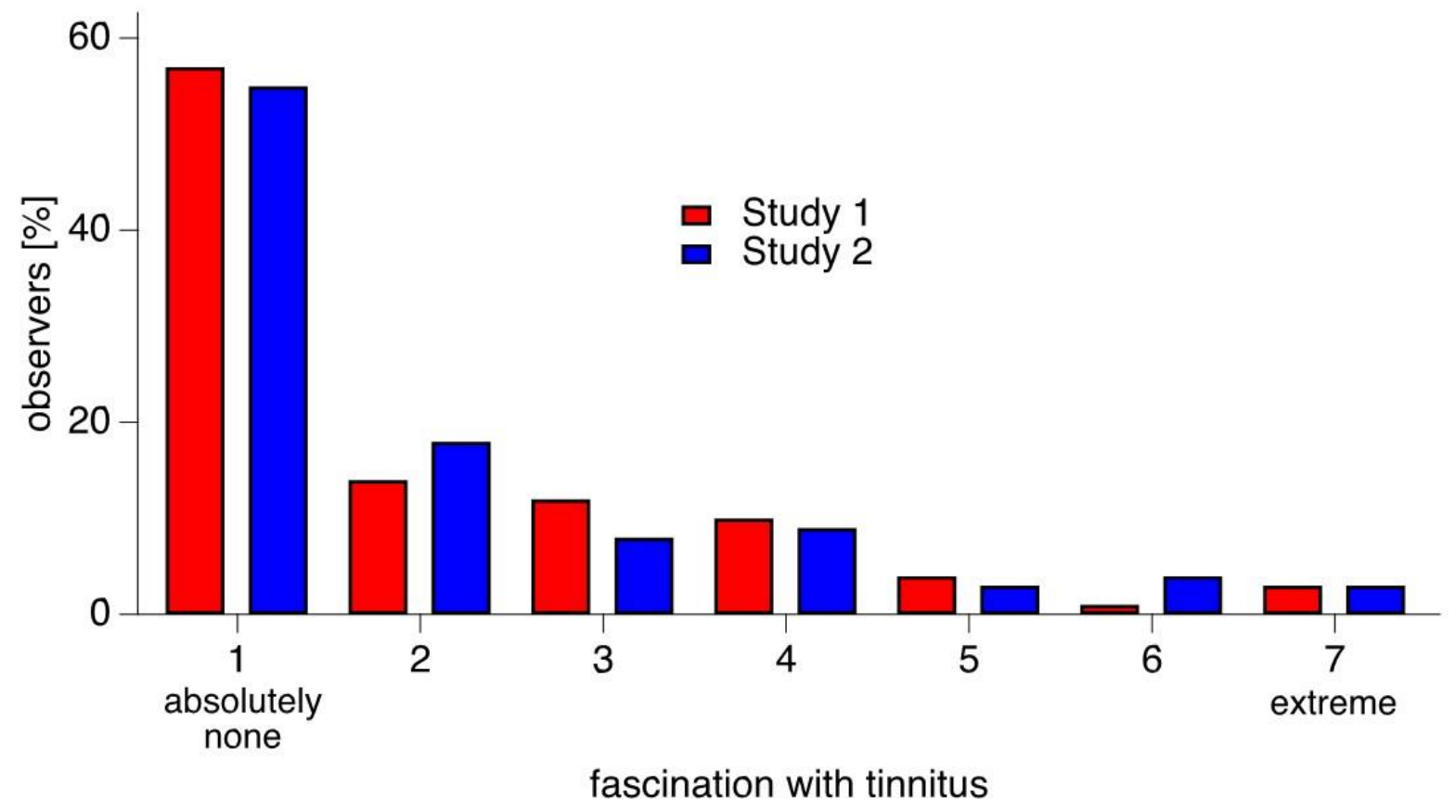

Figure 4. Distress and fascination with tinnitus. 


\section{Appendix}

Descriptive statistics for migraine, low blood pressure, blood pressure drops, ophthalmological problems, screen time and lifetime use of psychoactive substances.

\begin{tabular}{|l|c|}
\hline & $\%$ \\
\hline Migraine (Study 1) & 29.8 \\
\hline Migraine (Study 2) & 33.7 \\
\hline Ophthalmological problems (Study 1) & 53.1 \\
\hline Ophthalmological problems (Study 2) & 24.0 \\
\hline Low blood pressure & 23.5 \\
\hline Blood pressure drops & 45.4 \\
\hline Lifetime use of cannabis. & 9.6 \\
\hline Lifetime use of psychedelics & 13.1 \\
\hline Lifetime use of MDMA & 12.7 \\
\hline Lifetime use of cocaine/stimulants & 45.8 \\
\hline Lifetime use of any the psychoactive substances & \\
\hline listed above & \\
\hline
\end{tabular}


Frequency of tinnitus.

\begin{tabular}{|l|c|c|}
\hline & Study 1 & Study 2 \\
\hline Estimated percentage of & $\mathrm{N}=482$ & $\mathrm{~N}=277$ \\
time with tinnitus & & \\
$0 \%$ & 41.9 & 52.0 \\
$10 \%$ & 18.3 & 10.5 \\
$20 \%$ & 9.1 & 9.0 \\
$30 \%$ & 7.1 & 4.3 \\
$40 \%$ & 3.7 & 6.1 \\
$50 \%$ & 5.0 & 3.2 \\
$60 \%$ & 3.1 & 4.0 \\
$70 \%$ & 2.1 & 4.0 \\
$80 \%$ & 2.5 & 2.9 \\
$90 \%$ & 3.1 & 1.1 \\
$100 \%$ & 4.1 & 2.9 \\
\hline
\end{tabular}


Frequency of floaters and blue field entoptic phenomena.

\begin{tabular}{|l|c|l|c|}
\hline & Study 1 & Study 1 \\
\hline $\begin{array}{l}\text { Estimated } \\
\text { percentage of time } \\
\text { seeing floaters }\end{array}$ & $\mathrm{N}=450$ & $\begin{array}{l}\text { Estimated percentage } \\
\text { of time seeing blue field } \\
\text { entoptic phenomenon }\end{array}$ & $\mathrm{N}=456$ \\
$0 \%$ & 46.7 & $0 \%$ & 66.4 \\
$10 \%$ & 18.2 & $10 \%$ & 12.1 \\
$20 \%$ & 8.2 & $20 \%$ & 4.4 \\
$30 \%$ & 4.7 & $30 \%$ & 3.9 \\
$40 \%$ & 4.7 & $40 \%$ & 1.3 \\
$50 \%$ & 5.3 & $50 \%$ & 2.4 \\
$60 \%$ & 2.7 & $60 \%$ & 1.1 \\
$70 \%$ & 1.8 & $70 \%$ & 1.3 \\
$80 \%$ & 1.8 & $80 \%$ & .9 \\
$90 \%$ & .4 & $90 \%$ & 1.1 \\
$100 \%$ & 5.6 & $100 \%$ & 5.0 \\
\hline
\end{tabular}

